\title{
Open heavy-flavour production from small to large collision systems with ALICE at the LHC
}

\author{
Fabio Catalano ${ }^{\dagger, *}$ \\ Politecnico di Torino, \\ Corso Duca degli Abruzzi 24, Torino, 10129, Italy \\ INFN sez. Torino, \\ Via Pietro Giuria 1, Torino, 10125, Italy \\ E-mail: fabio.catalano@cern.ch
}

Heavy quarks are effective probes of the hot and dense nuclear matter, the quark-gluon plasma, produced in ultra-relativistic heavy-ion collisions. Due to the short time scale characterising their production, heavy quarks experience the whole evolution of the system. In particular, measurements of heavy-flavour hadron production in $\mathrm{Pb}-\mathrm{Pb}$ collisions at $\mathrm{LHC}$ energies give insight into the mechanisms of heavy-quark transport in the deconfined matter. In small hadronic systems, $\mathrm{pp}$ and $\mathrm{p}-\mathrm{Pb}$ collisions, heavy-flavour measurements provide the baseline for observations of hotmedium effects in heavy-ion collisions, as well as tests of perturbative quantum chromodynamic calculations and measurements of cold-nuclear-matter effects. In this contribution, recent ALICE results on open heavy-flavour hadron production in $\mathrm{pp}, \mathrm{p}-\mathrm{Pb}$ and $\mathrm{Pb}-\mathrm{Pb}$ collisions at various energies are discussed. New measurements are presented both for fully-reconstructed charmed hadrons and for single electrons from heavy-flavour hadron decays, also investigating the beautyquark production via the measurement of $\mathrm{D}$ mesons and electrons from beauty-hadron decays.

HardProbes 2020

1-6 June 2020

Austin, Texas

\footnotetext{
*Speaker

$\dagger$ on behalf of the ALICE Collaboration
} 


\section{Introduction}

Due to their large masses, charm and beauty quarks (heavy quarks) are mainly produced in hard-scattering processes between partons of the colliding nucleons. Therefore, their production can be described by perturbative quantum chromodynamic (pQCD) calculations down to zero transverse momentum $\left(p_{\mathrm{T}}\right)$. In ultra-relativistic heavy-ion collisions extreme temperatures are reached and lattice QCD calculations predict a phase transition of nuclear matter to a colourdeconfined medium, called quark-gluon plasma (QGP). Heavy quarks are produced in the initial stages of the nucleus-nucleus $(\mathrm{Pb}-\mathrm{Pb})$ collision, before the QGP formation, and experience the full evolution of the system while propagating through the medium and strongly interacting with the QGP constituents. In $\mathrm{Pb}-\mathrm{Pb}$ collisions the measurement of hadrons containing heavy quarks provides crucial information on the in-medium parton energy loss. The comparison of heavyflavour and light-flavour hadrons gives insight into the colour-charge and quark-mass dependence of energy loss. Moreover, the hadronisation mechanism of heavy quarks in the medium can be investigated comparing the production of different heavy-flavour hadron species, e.g., charmed baryons and mesons, or hadrons with and without strange-quark content [1]. Measurements of open heavy-flavour hadron production in proton-proton collisions are relevant tests of pQCD model calculations.

In ALICE, charmed hadrons are reconstructed at midrapidity $(|y|<0.8)$ via the hadronic decay channels: $\mathrm{D}^{0} \rightarrow \mathrm{K}^{-} \pi^{+}, \mathrm{D}^{+} \rightarrow \mathrm{K}^{-} \pi^{+} \pi^{+}, \mathrm{D}^{*+} \rightarrow \mathrm{D}^{0} \pi^{+} \rightarrow \mathrm{K}^{-} \pi^{+} \pi^{+}, \mathrm{D}_{\mathrm{s}}^{+} \rightarrow \phi \pi^{+} \rightarrow \mathrm{K}^{-} \mathrm{K}^{+} \pi^{+}$, $\Lambda_{\mathrm{c}}^{+} \rightarrow \mathrm{pK}_{\mathrm{s}}^{0}$ and their charge conjugates. Particle candidates are built from pairs or triplets of tracks with the proper charge combination. Kinematic and geometrical selections on the displaced decay-vertex topology, together with particle identification, are applied to reduce the combinatorial background. The charmed-hadron raw yields are obtained from an invariant-mass analysis and the reconstruction efficiencies are estimated using Monte Carlo simulations [2]. In addition, heavy-flavour hadrons are studied through the measurement of electrons produced in their semileptonic decays. Electrons are identified at midrapidity using the information provided by ALICE central-barrel detectors [3]. The hadron contamination and electrons from non-heavy-flavour sources, mainly photon conversions and Dalitz decays of light neutral mesons, are subtracted from the measured inclusive yield, which is then corrected for the acceptance and selection efficiency [4]. Exclusive measurements of prompt charmed hadrons, originated from the hadronisation of charm quarks produced in the initial collision, and non-prompt ones, which are produced from beauty-hadron decays, are possible thanks to the longer proper mean-life of hadrons containing beauty quarks. This allows the ALICE experiment to assess beauty-quark production through the measurement of non-prompt $\mathrm{D}$ mesons and electrons from beauty-hadron decays.

\section{Open heavy-flavour production in pp collisions}

The production cross section of prompt [2] and non-prompt D mesons and of electrons from semi-leptonic heavy-flavour hadron decays [4] is measured at midrapidity in pp collisions at $\sqrt{s}=$ $5.02 \mathrm{TeV}$. In the left panel of Fig. 1, prompt and new measurements of non-prompt $\mathrm{D}^{+}$mesons are compared, respectively, to FONLL [5] predictions and to FONLL with the B $\rightarrow \mathrm{D}+\mathrm{X}$ decay kinematics described by the PYTHIA8 package [6]. In the right panel, the cross section of 
heavy-flavour decay electrons is compared to theoretical calculations. The measurements are well
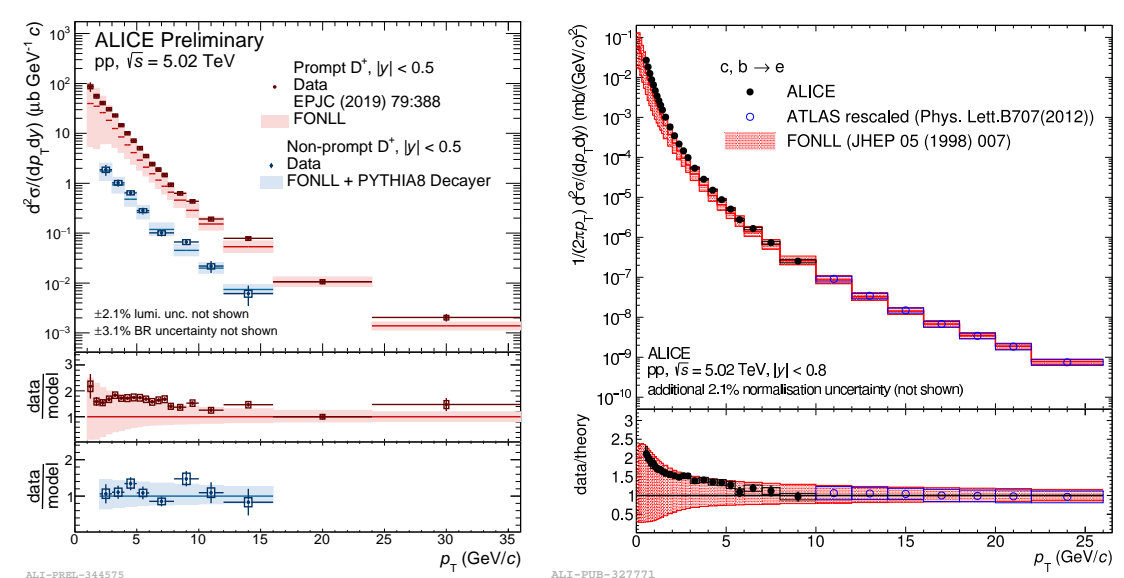

Figure 1: Left: prompt and non-prompt $\mathrm{D}^{+}$-meson cross sections in pp collisions at $\sqrt{s}=5.02 \mathrm{TeV}$ compared to FONLL predictions. Right: $\mathrm{e}^{ \pm}$from heavy-flavour hadron decays compared to FONLL predictions.

described by $\mathrm{pQCD}$ calculations. The prompt $\mathrm{D}^{+}$-meson measurement lies on the upper part of the FONLL prediction uncertainty band, while non-prompt $\mathrm{D}^{+}$mesons are in good agreement with the central predictions. A similar behaviour is observed for heavy-flavour decay electrons, where the cross section is on the upper edge of FONLL predictions for $p_{\mathrm{T}}<5 \mathrm{GeV} / c$ and moves towards the central values at high $p_{\mathrm{T}}$, where beauty-hadron decays are the dominant contribution.

\section{3. $D_{\mathrm{s}}^{+}$-meson abundance as a function of particle multiplicity}

Figure 2 shows the yield ratio between $\mathrm{D}_{\mathrm{s}}^{+}$and $\mathrm{D}^{0}$ mesons measured by the ALICE experiment in $\mathrm{p}-\mathrm{Pb}$ [7] and $\mathrm{Pb}-\mathrm{Pb}$ [8] collisions at $\sqrt{s_{\mathrm{NN}}}=5.02 \mathrm{TeV}$, as a function of the charged-particle multiplicity for different $p_{\mathrm{T}}$ intervals, together with new measurements in pp at $\sqrt{s}=13 \mathrm{TeV}$ and in $\mathrm{Pb}-\mathrm{Pb}$ collisions. The $\mathrm{D}_{\mathrm{s}}^{+} / \mathrm{D}^{0}$ ratio in pp collisions does not show a dependence on the event multiplicity and it is in agreement with the expected value considering the charm-quark fragmentation fractions measured in $\mathrm{e}^{+} \mathrm{e}^{-}$collisions at LEP [9]. The measurement in minimum bias $\mathrm{p}-\mathrm{Pb}$ collisions is compatible to what is observed in pp collisions at similar multiplicity values. An increase of the $\mathrm{D}_{\mathrm{s}}^{+} / \mathrm{D}^{0}$ ratio with respect to $\mathrm{pp}$ and $\mathrm{p}-\mathrm{Pb}$ is observed in $\mathrm{Pb}-\mathrm{Pb}$ collisions at $p_{\mathrm{T}}<8 \mathrm{GeV} / c$. This higher production of $\mathrm{D}_{\mathrm{s}}^{+}$mesons in $\mathrm{Pb}-\mathrm{Pb}$ is expected if charm quarks hadronise via coalescence with a quark of the QGP medium, where the production of ss pairs is enhanced [1].

\section{Open heavy-flavour nuclear modification factor}

The production of prompt charmed hadrons and electrons from heavy-flavour hadron decays [4] is measured in $\mathrm{Pb}-\mathrm{Pb}$ collisions at $\sqrt{s_{\mathrm{NN}}}=5.02 \mathrm{TeV}$ and compared to pp collisions through the nuclear modification factor $R_{\mathrm{AA}}\left(p_{\mathrm{T}}\right)=\left(\mathrm{d} N_{\mathrm{AA}} / \mathrm{d} p_{\mathrm{T}}\right) /\left(\left\langle N_{\text {coll }}^{\mathrm{AA}}\right\rangle \cdot \mathrm{d} N_{\mathrm{pp}} / \mathrm{d} p_{\mathrm{T}}\right)$; where $\mathrm{d} N_{\mathrm{AA}} / \mathrm{d} p_{\mathrm{T}}$ and $\mathrm{d} N_{\mathrm{pp}} / \mathrm{d} p_{\mathrm{T}}$ are the $p_{\mathrm{T}}$-differential yields measured in nucleus-nucleus and pp collisions, respectively, and $\left\langle N_{c o l l}^{\mathrm{AA}}\right\rangle$ is the average number of binary interactions in a nucleus-nucleus collision. In the left 


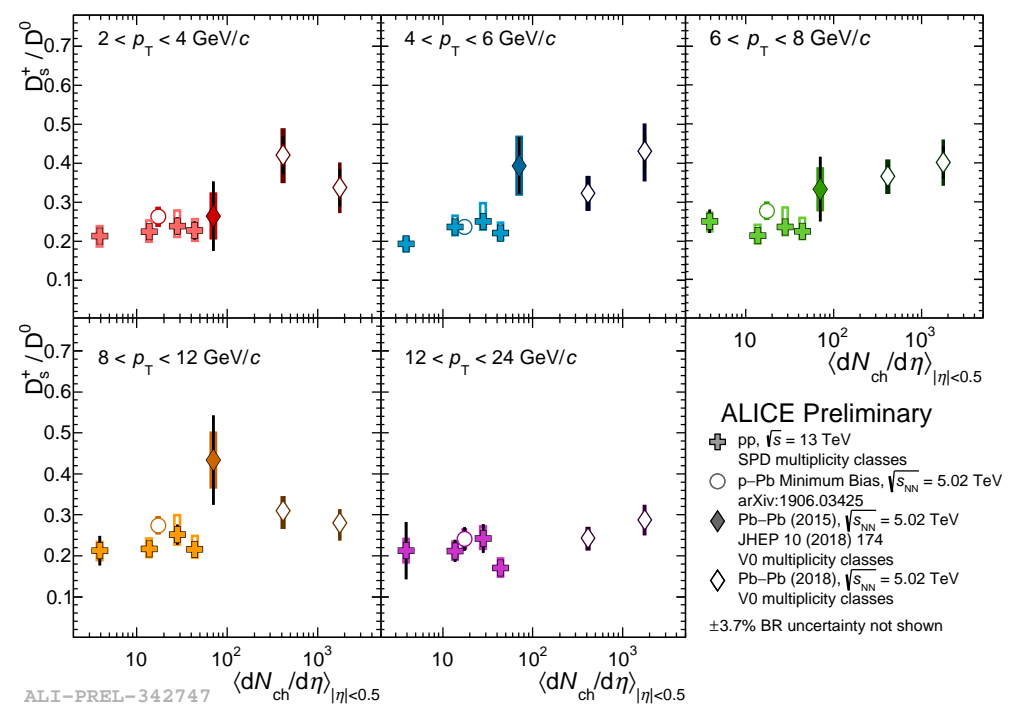

Figure 2: $\mathrm{D}_{\mathrm{s}}^{+} / \mathrm{D}^{0}$ ratio measured in $\mathrm{pp}, \mathrm{p}-\mathrm{Pb}$ and $\mathrm{Pb}-\mathrm{Pb}$ collisions as a function of the charged-particle multiplicity and for different $p_{\mathrm{T}}$ intervals.

panel of Fig. 3, the measured $R_{\mathrm{AA}}$ of strange and non-strange D mesons, $\Lambda_{\mathrm{c}}^{+}$and charged particles [10] in central $\mathrm{Pb}-\mathrm{Pb}$ collisions are reported. A strong suppression of the charmed and light-flavour
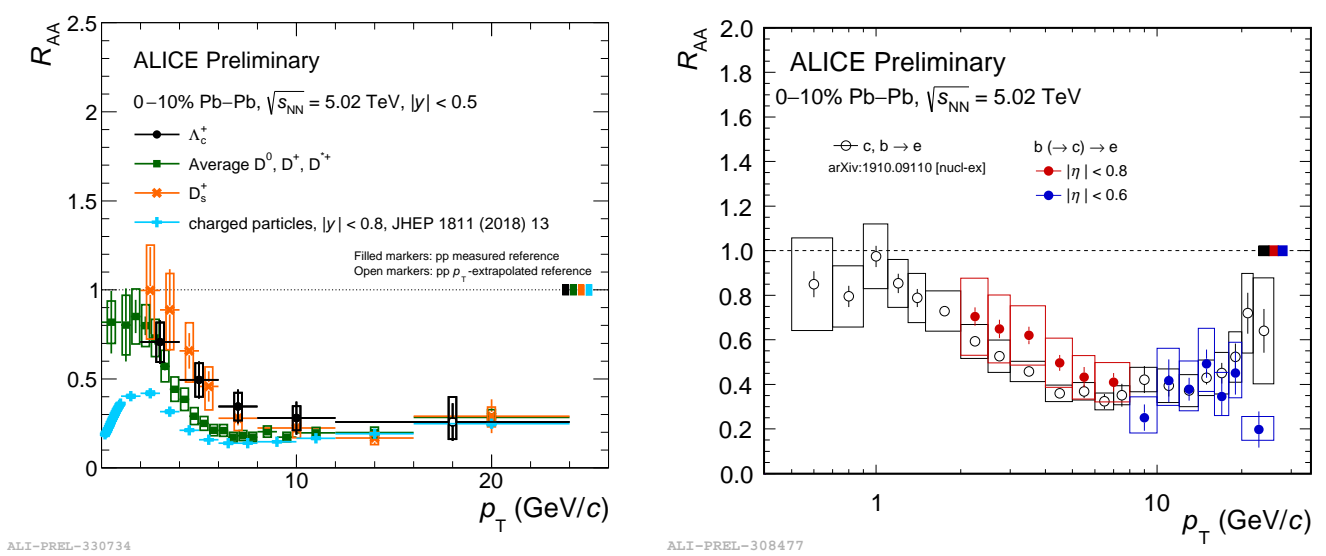

Figure 3: Left: $R_{\mathrm{AA}}$ comparison between $\Lambda_{\mathrm{c}}^{+}$, non-strange $\mathrm{D}$ mesons, $\mathrm{D}_{\mathrm{s}}^{+}$and charged particles in the $0-10 \%$ centrality class. Right: $R_{\mathrm{AA}}$ comparison of $\mathrm{e}^{ \pm}$from heavy-flavour hadron and beauty-hadron decays.

hadron $R_{\mathrm{AA}}$ is observed, as expected in the presence of the QGP due to in-medium energy loss. The $R_{\mathrm{AA}}$ of non-strange $\mathrm{D}$ mesons is higher than that of charged particles below $4 \mathrm{GeV} / c$, while they are compatible at higher $p_{\mathrm{T}}$. This behaviour can be explained by the mass and colour-charge dependence of energy loss. However, also other factors play a role, such as the different initial $p_{\mathrm{T}}$ distributions and fragmentation functions of charm and light quarks and the different effects of hadronisation via recombination and radial flow [8]. Finally, there is an indication of a smaller suppression of $\mathrm{D}_{\mathrm{s}}^{+}$mesons and $\Lambda_{\mathrm{c}}^{+}$baryons than non-strange $\mathrm{D}$ mesons at $p_{\mathrm{T}}<8 \mathrm{GeV} / c$. In the right panel of Fig. 3, the $R_{\mathrm{AA}}$ of electrons from semi-leptonic beauty-hadron decays in central $\mathrm{Pb}-\mathrm{Pb}$ collisions is compared to that of inclusive heavy-flavour decay electrons. The $R_{\mathrm{AA}}$ of electrons 
coming from beauty hadrons is above the inclusive one in all the $p_{\mathrm{T}}$ intervals below $10 \mathrm{GeV} / c$, even if compatible within uncertainties, pointing to a possible mass dependence of heavy-quark energy loss, where beauty quarks lose less energy in the medium than charm quarks.

\section{Conclusions}

The ALICE collaboration has measured the production of charmed hadrons and electrons from heavy-flavour hadron decays in different collision systems and at different centre-of-mass energies. In addition, the production of beauty quarks has been investigated with the measurement of non-prompt $\mathrm{D}$ mesons and electrons from beauty-hadron decays.

In pp collisions, the measured D-meson and heavy-flavour electron cross sections are compatible with $\mathrm{pQCD}$ calculations. In $\mathrm{Pb}-\mathrm{Pb}$ collisions, the indication of a higher $\mathrm{D}_{\mathrm{s}}^{+} / \mathrm{D}^{0}$ ratio than in $\mathrm{pp}$, at low $p_{\mathrm{T}}$, is in agreement with the charm-quark hadronisation via quark recombination in the QGP. The D-meson $R_{\mathrm{AA}}$ is higher than that of charged particles at $p_{\mathrm{T}}<4 \mathrm{GeV} / c$, suggesting a colour-charge and quark-mass dependence of the energy loss. Furthermore, an indication of a smaller energy loss of beauty quarks than charm quarks is observed through the measurement of electrons from beauty-hadron decays.

\section{References}

[1] I. Kuznetsova and J. Rafelski, "Heavy flavor hadrons in statistical hadronization of strangeness-rich QGP,” Eur. Phys. J. C51 (2007) 113-133, hep-ph/0607203.

[2] ALICE Collaboration, S. Acharya et. al., "Measurement of $\mathrm{D}^{0}, \mathrm{D}^{+}, \mathrm{D}^{*+}$ and $\mathrm{D}_{\mathrm{s}}^{+}$production in pp collisions at $\sqrt{s}=5.02 \mathrm{TeV}$ with ALICE," Eur. Phys. J. C79 (2019), no. 5 388, 1901.07979.

[3] ALICE Collaboration, K. Aamodt et. al., "The ALICE experiment at the CERN LHC," JINST 3 (2008) S08002.

[4] ALICE Collaboration, S. Acharya et. al., "Measurement of electrons from semileptonic heavy-flavour hadron decays at midrapidity in pp and Pb-Pb collisions at $\sqrt{s_{\mathrm{NN}}}=5.02 \mathrm{TeV}$," Phys. Lett. B 804 (2020) 135377, 1910.09110.

[5] M. Cacciari, M. Greco, and P. Nason, "The P(T) spectrum in heavy flavor hadroproduction," JHEP 05 (1998) 007, hep-ph/9803400.

[6] T. Sjöstrand et. al., "An introduction to PYTHIA 8.2," Comput. Phys. Commun. 191 (2015) 159-177, 1410.3012.

[7] ALICE Collaboration, S. Acharya et. al., "Measurement of prompt $\mathrm{D}^{0}, \mathrm{D}^{+}, \mathrm{D}^{*+}$, and $\mathrm{D}_{\mathrm{s}}^{+}$production in p-Pb collisions at $\sqrt{\mathrm{s}_{\mathrm{NN}}}=5.02 \mathrm{TeV}$," JHEP 12 (2019) 092, 1906.03425.

[8] ALICE Collaboration, S. Acharya et. al., "Measurement of $\mathrm{D}^{0}, \mathrm{D}^{+}, \mathrm{D}^{*+}$ and $\mathrm{D}_{s}^{+}$production in $\mathrm{Pb}-\mathrm{Pb}$ collisions at $\sqrt{\mathrm{s}_{\mathrm{NN}}}=5.02 \mathrm{TeV}$, JHEP 10 (2018) 174, 1804.09083 .

[9] L. Gladilin, "Fragmentation fractions of $c$ and $b$ quarks into charmed hadrons at LEP," Eur. Phys. J. C 75 (2015), no. 1 19, 1404.3888.

[10] ALICE Collaboration, S. Acharya et. al., "Transverse momentum spectra and nuclear modification factors of charged particles in pp, p-Pb and Pb-Pb collisions at the LHC," JHEP 11 (2018) 013, 1802.09145 . 